\title{
Association between levobupivacaine and pancuronium. Interference in neuromuscular transmission and blockade in rats ${ }^{1}$
}

\author{
Vanessa Henriques Carvalho ${ }^{\mathrm{I}}$, Angélica de Fátima de Assunção Braga ${ }^{\mathrm{II}}$, Franklin Sarmento da Silva Braga ${ }^{\mathrm{III}}$, Gloria Maria \\ Braga Potério $^{\mathrm{IV}}$, Filipe Nadir Caparica Santos ${ }^{\mathrm{v}}$, Fernando Eduardo Féres Junqueira ${ }^{\mathrm{VI}}$
}

DOI: http://dx.doi.org/10.1590/S0102-865020160070000009

${ }^{I} \mathrm{PhD}$, Department of Anesthesiology, Faculty of Medical Sciences, Universidade Estadual de Campinas (UNICAMP), Brazil. Intellectual, scientific and design of the study; acquisition and interpretation of data; technical procedures; manuscript preparation and writing, critical revision, supervised all phases of the study.

IIAssociate Professor, Department of Anesthesiology, Faculty of Medical Sciences, UNICAMP, Campinas-SP, Brazil. Intellectual, scientific and design of the study; interpretation of data; technical procedures; manuscript preparation and writing, critical revision, supervised all phases of the study.

IIIPhD, Department of Anesthesiology, Faculty of Medical Sciences, UNICAMP, Campinas-SP, Brazil. Critical revision.

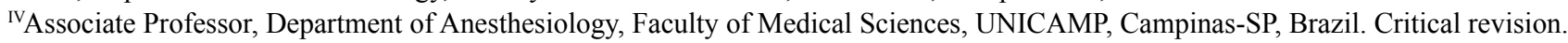

${ }^{v}$ MD, Department of Anesthesiology, School of Medicine, UNICAMP, Campinas-SP, Brazil. Acquisition of data.

${ }^{\mathrm{V}}$ Resident, Department of Anesthesiology, School of Medicine, UNICAMP, Campinas-SP, Brazil. Acquisition of data.

\section{ABSTRACT}

PURPOSE: To evaluate the effects of levobupivacaine on neuromuscular transmission and neuromuscular blockade produced by pancuronium in vitro.

METHODS: Thirty rats were distributed into groups $(\mathrm{n}=5)$ according to the drug used alone or in combination: Group I - levobupivacaine (5 $\left.\mu \mathrm{g} \cdot \mathrm{mL}^{-1}\right)$; Group II - pancuronium $\left(2 \mu \mathrm{g} \cdot \mathrm{mL}^{-1}\right)$; Group III - pancuronium $\left(2 \mu \mathrm{g} \cdot \mathrm{mL}^{-1}\right)+$ levobupivacaine $\left(5 \mu \mathrm{g} \cdot \mathrm{mL}^{-1}\right)$. The following parameters were evaluated: 1) amplitude of diaphragmatic response to indirect stimulation, before and 60 minutes after the addition of levobupivacaine and pancuronium alone, and after the addition of levobupivacaine combined with pancuronium; 2) membrane potentials (MP) and miniature endplate potentials (MEPP).

RESULTS: Levobupivacaine alone did not alter the amplitude of muscle response and MP. In preparations previoulsy exposed to levobupivacaine, the block with pancuronium was significantly denser $(90.2 \pm 15.2 \%)$, showing a significant difference $(\mathrm{p}=0.031)$ in comparison to the block produced by pancuronium alone $(48.9 \% \pm 9.8 \%)$. There was a decrease in the frequency and amplitude of MEPPs.

CONCLUSION: Levobupivacaine potentiated the neuromuscular blockade produced by pancuronium, confirming a presynaptic action by a decrease in miniature endplate potentials.

Key words: Pancuronium. Neuromuscular Nondepolarizing Agents. Rats. 


\section{Introduction}

To explain the interaction between local anesthetics and neuromuscular blocking agents, several mechanisms have been described, including a presynaptic action by inhibition of acetylcholine release, a postsynaptic action due to stabilization of the postjunctional membrane, and interference with the phenomenon of excitation-contraction coupling of the muscle fiber ${ }^{1-4}$. Although only high concentrations of local anesthetics can produce neuromuscular blockade, the interaction between these drugs and nondepolarizing neuromuscular blocking agents in particular is relevant in clinical practice, when both groups of drugs are used simultaneously and careful patient monitoring is required. Local anesthetics, especially amino amides, are drugs commonly used by different routes in anesthetic practice, either alone or in combination with general anesthesia ${ }^{5-7}$.

With the advent of stereoisomerism in local anesthetics, a major advance was made in pharmacological research mainly related to safety issues concerning drug toxicity. Levobupivacaine is a $100 \%$ (S-) pure enantiomer of racemic bupivacaine (S50-R50) that has a lower lipid solubility, lower cardiotoxicity and neurotoxicity, a greater sensory blockade and less profound motor blockade than racemic bupivacaine ${ }^{4,6}$. Pancuronium is a longacting aminosteroid, nondepolarizing neuromuscular blocking agent, used in prolonged surgeries and in intensive care units ${ }^{1}$. It is fundamental to identify the interaction between these drugs, since the duration of their effects may be prolonged. The margin of safety of the neuromuscular junction may be impaired, leading to a higher risk of residual blockade and postoperative complications. The aim of this study was to evaluate the effect of levobupivacaine on neuromuscular transmission in an experimental model and its influence on the neuromuscular block produced by pancuronium.

\section{Methods}

Procedures were performed in compliance with the ethical principles of animal experimentation adopted by the Brazilian College of Animal Experimentation (COBEA). Study protocol was approved by the Ethics Committee in Animal Experimentation of the Institute of Biology of the Universidade Estadual de Campinas (protocol no 2346-1).

Thirty male Wistar rats were used (weight ranging from 180 to $250 \mathrm{~g}$ ). The animals were anesthetized with urethane (1.2mg. $\mathrm{kg}^{-1}$ by the intraperitoneal route) and exsanguinated by sectioning of the neck blood vessels, to facilitate the identification and removal of the left hemidiaphragm and part of the corresponding phrenic nerve. The hemidiaphragms with corresponding phrenic nerves were fixed in a vat containing 40 $\mathrm{mL}$ of Tyrode's solution, aired constantly with carbogen $(95 \%$ $\mathrm{O}_{2}+5 \% \mathrm{CO}_{2}$ ) and maintained at $37^{\circ} \mathrm{C}$. The preparation was obtained from rat muscle according to a technique described by Bulbring ${ }^{8}$. The nerve was placed on platinum electrodes connected to a Grass S48 stimulator. The diaphragm was maintained by its tendinous portion under constant tension $(5.0 \mathrm{~g})$ through a wire attached to a Load Cell BG50 GMS isometric transducer. Indirect stimulation of the diaphragm was performed at a frequency of $0.1 \mathrm{~Hz}$ and duration of $0.2 \mathrm{mseg}$. Variations in tension produced by diaphragmatic contractions were recorded by a Gould RS 3400 physiographer. . In a previous experimental study ${ }^{1}$, it was observed that the degree of motor blockade was significantly greater in rats exposed to the combination of local anesthetic + pancuronium $(93.8 \%$, ) in comparison to those exposed only to the neuromuscular blocking agent $(54.9 \%)$, with a difference of $38.9 \%$. Studies evaluating the effects of levobupivacaine on the neuromuscular block produced by pancuronium were not found in the literature. Therefore, for sample calculation, we employed the variable degree of motor blockade. Considering arbitrarily an increase in approximately $85 \%$ of animals exposed simultaneously to LA and neuromuscular blocking agent, in comparison to nonexposed rats, with a significant difference, beta error $=20 \%$ (power $=80 \%$, alpha error $=5 \%$, and ratio of $1: 1$ between exposed and non-exposed animals, we achieved a sample size of five rats per group. The distribution of rats in the 3 groups was determined by lot: Group I - levobupivacaine $\left(5 \mu \mathrm{g} . \mathrm{mL}^{-1}\right)$; Group II - pancuronium $\left(2 \mu \mathrm{g} \cdot \mathrm{mL}^{-1}\right)$; Group III - pancuronium $\left(2 \mu \mathrm{g} \cdot \mathrm{mL}^{-1}\right)$ in a preparation previously exposed to levobupivacaine $\left(5 \mu \mathrm{g} \cdot \mathrm{mL}^{-1}\right)$. In Group III, pancuronium was added to the preparation 30 minutes after the addition of levobupivacaine. The study drugs were provided by the Cristália Laboratory. Muscle response to indirect stimulation was recorded during 60 minutes after the addition of the drugs. In the rat diaphragm, the effects of levobupivacaine on miniature end-plate potentials and membrane potentials were also studied. To capture MEPPs, a glass microelectrode was implanted on or as close as possible to the region of the motor end-plate, with the aid of a micromanipulator (Leitz). Biopotentials were obtained by means of a signal amplifier (Getting Microelectrode Amplifier, MA, USA) and observed in a Tektronix oscilloscope. MEPP recordings were made in a microcomputer (Microtec, São Paulo-SP) running with software for data acquisition (AqDados, Lynx, São Paulo-SP). These were recorded before (control), at 30 and 60 minutes after the addition of levobupivacaine. To determine membrane potential, with the aid of a microscope, a microelectrode was inserted 
intracellularly on superficial muscle fibers (at distant regions of the motor endplate), measuring the vertical displacement suffered by the oscilloscope beam, at the time of insertion. Five membrane potentials were measured at each time point determined, obtaining a mean value. At time zero (control), the potentials were measured before the addition of levobupivacaine. After this measurement, the local anesthetic at a concentration of $5 \mu \mathrm{g} / \mathrm{mL}$ was added to the bath and readings at 10, 20,30, 40, 50 and 60 minutes were taken. Five experiments were performed, calculating and the arithmetic means and standard deviations of the readings obtained.

The following parameters were assessed: 1) the amplitude of diaphragmatic response to indirect stimulation, before and 60 minutes after the addition of levobupivacaine; 2) the amplitude of diaphragmatic response to indirect stimulation, before and 60 minutes after the addition of pancuronium alone and in a preparation previously exposed to levobupivacaine; 3 ) membrane potentials (MP) and miniature endplate potentials (MEPS). The results were expressed in means and standard deviations. For statistical analysis, Student's t test was used for the comparative evaluation of the degree of motor blockade produced by the combination of levobupivacaine and pancuronium in relation to the block produced by pancuronium alone. The Wilcoxon test was used for paired samples in membrane potentials. A significance level of $5 \%$ was adopted $(\mathrm{p}<0.05)$. The power of the test was calculated and $\beta>20 \%$ was obtained (power $>80 \%$ ).

\section{Results}

Levobupivacaine alone and used in the concentration studied in a rat phrenic nerve-diaphragm preparation did not cause a reduction in the amplitude of muscle response to indirect electrical stimulation. In preparations that had been previously exposed to levobupivacaine, the block produced by pancuronium was $90.2 \pm 15.2 \%$, with a significant difference $(\mathrm{p}=0.031)$, compared to the block produced by pancuronium alone $(48.9 \% \pm$ 9.8\%). Membrane potentials did not show any significant changes in different time points of evaluation, ranging from 77.20 to $80.24 \mathrm{mV}$. The effects of levobupivacaine on miniature endplate potentials (MEPPs) were evidenced by a decrease greater than $60 \%$ in frequency and amplitude, at 30 minutes of exposure to the drug, followed by complete disappearance at 60 minutes.

\section{Discussion}

The current study conducted in a rat phrenic nervediaphragm preparation, showed that in the concentration studied levobupivacaine used alone, did not exert any effects on the neuromuscular junction. However, it potentiated the block produced by pancuronium.

The interaction and potentiation of the effects of neuromuscular blocking agents by local anesthetics, that was previously observed and described in experimental and clinical studies, is of great importance ${ }^{1-3,5-7}$. Nevertheless, experimental studies performed in isolated preparations permit the exclusion of bias, which may occur in clinical trials. The wide individual variability observed with the drugs used and other factors present during general anesthesia, inherent to the patient, anesthetics, drugs administered in the perioperative period and alterations including hypothermia, acid-base disturbances can bias the results of the study ${ }^{1-3,5,6}$.

In clinical practice, the association between anesthetic techniques and the use of local anesthetics has become increasingly common. The administration of local anesthetics may reach considerable plasma levels, leading to impairment of neuromuscular transmission. In a study conducted with a preparation similar to ours, it was shown that ED50 of d-tubocurarine was significantly decreased by different local anesthetics that were used in concentrations considered ineffective. This result expressed an increased potentiation of the neuromuscular blocking agent ${ }^{9}$. These findings, which were also observed in other experimental studies, may infer that the interaction between neuromuscular blocking agents and local anesthetics may be due to real potentiation caused by the action of both classes of drugs, at different sites of the neuromuscular junction ${ }^{1-4,9}$.

The influence of $50 \%$ enantiomeric excess bupivacaine mixture and ropivacaine on the pharmacodynamic characteristics of pancuronium was evaluated in experiments. An increase of about $100 \%$ and $90 \%$ in the degree of neuromuscular blockade, respectively, was observed, representing a significant statistical difference compared to the block produced by the use of pancuronium alone ${ }^{1,10}$.

There are no experimental research studies in the literature that have evaluated the association employed in this study. However, a clinical trial administering levobupivacaine by the epidural route, showed alterations in recovery from the block induced by vecuronium, a steroidal neuromuscular blocking agent, with a structure similar to pancuronium 6 . The authors described that the recovery index $(25-75 \% \mathrm{RI})$ and the time period for complete recovery $(\mathrm{T} 4: \mathrm{T} 1=90 \%)$ from the block produced by vecuronium were significantly longer $(\mathrm{p}<0.001)$ in patients receiving levobupivacaine, in comparison to those undergoing only general anesthesia. 
The interaction between local anesthetics and neuromuscular blocking agents is still not fully understood. The mechanism of action of these drugs seems to be multifactorial. Presynaptic and postsynaptic events have been described ${ }^{1-4,9,10}$. To identify the probable mechanism of interaction and potentiation of the block produced by pancuronium, in a preparation previously exposed to levobupivacaine, electrophysiological studies were conducted. In the evaluation of bioelectrical potentials, it was observed that levobupivacaine, in the concentration employed, did not alter the membrane potential of muscle fibers, which remained within normal limits $(-85 \mathrm{mV})$. It was demonstrated that the drug has no depolarizing action on skeletal muscle fibers, although it prevents the conduction of action potentials ${ }^{4}$.

The presynaptic action of levobupivacaine and its interference in the processes of production or release of the mediator, has been studied through evaluation of the miniature end-plate potentials, also termed plate minipotentials. These are small potentials with an amplitude corresponds to the hundredth part of the end-plate potential. The function of these potentials is unknown but appears to be related to the maintenance of muscle trophism. These electrophysiological events detect a small, continuous and spontaneous release of a single acetylcholine quantum, ineffective to trigger an action potential, without the need of any neuronal electrical stimulus. As previously described ${ }^{2-4}$ with other local anesthetics, it was also observed that levobupivacaine impairs neurotransmitter release, an event demonstrated by a progressive decrease in the frequency and amplitude of miniature endplate potentials, and decreased margin of safety of the neuromuscular junction. Therefore, these results may suggest that levobupivacaine has a presynaptic action in a manner similar to other local anesthetics, contributing to potentiation of the neuromuscular block produced by pancuronium.

\section{Conclusion}

Levobupivacaine potentiated the neuromuscular blockade produced by pancuronium, confirming a presynaptic action by a decrease in miniature endplate potentials.

\section{References}

1. Braga AF, Carvalho VH, Braga FS, Potério GM, Santos FN. Effect of ropivacaine combined with pancuronium on neuromuscular transmission and effectiveness of neostigmine and 4-aminopyridine for blockade reversal: experimental study. Rev Bras Anestesiol. 2015 Mar-Apr;65(2):136-40. doi: 10.1016/j.bjan.2013.10.020.

2. Braga AF, Carvalho VH, Braga FS, Rodrigues-Simioni L, Loyola YC, Potério GB. Influence of local anesthetics on the neuromuscular blockade produced by rocuronium: effects of lidocaine and 50\% enantiomeric excess bupivacaine on the neuromuscular junction. Rev Bras Anestesiol. 2009 Nov-Dec;59(6):725-34. PMID: 20011862.

3. Carvalho VH, Braga AF, Braga FS, Loyola YC, de Araújo DR, Mantovani $\mathrm{M}$. The influence of lidocaine and racemic bupivacaine on neuromuscular blockade produced by rocuronium. A study in rat phrenic nerve-diaphragm preparation. Acta Cir Bras. 2009 MayJun;24(3):211-5. PMID: 19504004.

4. Braga AF, Carvalho VH, Braga FS, Potério GM, Santos FN. Evidence of presynaptic and postsynaptic action of local anesthetics in rats. Acta Cir Bras. 2013 Nov;28(11):774-7. PMID: 24316744.

5. Suzuki T, Mizutani H, Ishikawa K, Miyake E, Saeki S, Ogawa S. Epidurally administered mepivacaine delays recovery of train-offour ratio from vecuronium-induced neuromuscular block. Br J Anaesth. 2007 Nov;99(5):721-5. PMID: 17855735.

6. Sahin SH, Colak A, Sezer A, Arar C, Sevdi S, Gunday I, Sut N. Effect of epidural levobupivacaine on recovery from vecuroniuminduced neuromuscular block in patients undergoing lower abdominal surgery. Anaesth Intensive Care. 2011 Jul;39(4):607-10. PMID: 21823377.

7. Cardoso LSM, Martins CR, Tardelli MA. Efeitos da lidocaína por via venosa sobre a farmacodinâmica do rocurônio. Rev Bras Anestesiol. 2005 Aug;55(4):371-80. PMID: 19468625.

8. Bulbring E. Observation on the isolated phyrenic nerve-diaphragm preparation of the rat. Br J Pharmacol Chemother. 1946 Mar;1:3861. PMID: 20999334.

9. Matsuo S, Rao DB, Chaudry I, Foldes FF. Interaction of muscle relaxants and local anesthetics at the neuromuscular junction. Anesth Analg. 1978 Sep-Oct;57(5):580-7. PMID: 568429.

10. de Assunção Braga AF, Carvalho VH, da Silva Braga FS, Potério GM, Santos FN, Junqueira FE. Effect of 50\% enantiomeric excess bupivacaine mixture combined with pancuronium on neuromuscular transmission in rat phrenic nerve-diaphragm preparation; a pilot study. Indian J Anaesth. 2015 Nov;59(11):701-5. doi: 10.4103/00195049.170019 .

\section{Correspondence:}

Vanessa Henriques Carvalho

Rua: Olympio Pattaro, 234

13085-045 Campinas - SP Brasil

Tel.: (55 19)3365-7315

vanessahcarvalho74@gmail.com

Received: Mar 20, 2016

Review: May 26, 2016

Accepted: Jun 24, 2016

Conflict of interest: none

Financial source: none

${ }^{1}$ Research performed at Laboratory of Neuromuscular Junction, Department of Pharmacology, Universidade Estadual de Campinas, Brazil. 